\title{
Nontuberculous mycobacteria in respiratory specimens: clinical significance at a tertiary care hospital in the north of Portugal
}

\author{
Micobactérias não tuberculosas em espécimes respiratórios: significado \\ clínico em um hospital terciário no norte de Portugal
}

\author{
Hans Dabó', Vanessa Santos', Anabela Marinho², Angélica Ramos ${ }^{3}$, \\ Teresa Carvalho ${ }^{3}$, Manuela Ribeiro ${ }^{3}$, Adelina Amorim ${ }^{2}$
}

\section{To the Editor:}

Nontuberculous mycobacteria (NTM) are ubiquitous in the human environment, and more than 150 NTM species have been described to date. ${ }^{(1)}$ When inhaled by susceptible individuals, such as those with chronic lung disease, NTM can lead to chronic, progressive, and sometimes fatal respiratory symptoms. Over the last three decades, the incidence of NTM laboratory isolation and related lung disease has been increasing, surpassing that of tuberculosis in some areas. ${ }^{(1,2)}$ However, the isolation of NTM from respiratory specimens might be due to contamination of specimens or colonization of patients that is transient, not necessarily indicating disease. ${ }^{(1)}$

Here, we report the incidence of NTM isolation in the Pulmonology Department of the São João Hospital Center, a tertiary care university hospital located in Porto, the second most populous city in Portugal. It is the largest hospital in the northern region and one of the three largest in the country. We retrospectively analyzed patients who were seronegative for HIV and for whom NTM had been isolated in at least one respiratory specimen between January of 2008 and December of 2012. The samples were decontaminated with the N-acetyl-L-cysteine-sodium hydroxide method and inoculated into Middlebrook 7H9 broth medium (BBL Mycobacteria Growth Indicator Tube [MGIT]; Becton Dickinson, Franklin Lakes, NJ, USA), in accordance with the manufacturer instructions. Positive cultures, incubated and monitored in an automated culture system (BACTEC MGIT 960; Becton Dickinson), were examined by smear microscopy with Kinyoun staining for AFB. In the presence of AFB, we used a molecular assay for common mycobacteria and additional species (GenoType Mycobacterium CM/ AS; Hain Lifescience GmbH, Nehren, Germany), in accordance with the manufacturer instructions, to determine the species of the isolates. Demographic, clinical, radiological, and microbiological data were collected. The clinical relevance of the isolation in the respiratory sample was defined in accordance with the current American Thoracic Society/Infectious Diseases Society of America (ATS/IDSA) criteria. ${ }^{(3)}$

Two hundred and two patients were included in this study. Of those, 118 (58\%) were male. The mean age was 64 years (range, $23-89$ years). The main risk factors identified were the underlying structural lung disease, such as COPD, in 73 patients $(36 \%)$, bronchiectasis, in $62(31 \%)$, and tuberculosis sequelae, in 40 (20\%); and nonpulmonary conditions, such as diabetes mellitus, in 18 patients (9\%), gastroesophageal reflux disease, in $16(8 \%)$, and receiving immunosuppression therapy, in 12 (6\%). Data on environmental exposure were not available. A total of 407 isolates were obtained, and the species were identified in 378 (93\%). Of the 407 isolates, 237 (58\%) were identified as being within the Mycobacterium avium complex (MAC) group and 141 (35\%) were identified as belonging to one of 11 other Mycobacterium species, the remaining $29(7 \%)$ being identified as mycobacteria but not down to the species level (Table 1). These isolates were obtained from sputum samples in 192 (95\%) of the patients, bronchial lavage fluid samples in $13(6 \%)$, BAL fluid samples in 7 (4\%), a lung biopsy sample in $1(0.5 \%)$, and a gastric aspirate sample in 1

1. Intern in the Department of Pulmonology, São João Hospital Center, EPE, Porto, Portugal.

2. Attending Physician. Department of Pulmonology, São João Hospital Center, EPE, Porto, Portugal.

3. Attending Physician. Department of Clinical Pathology, São João Hospital Center, EPE, Porto, Portugal. 
Table 1 - Nontuberculous mycobacteria isolated from respiratory specimens collected from 202 patients.

\begin{tabular}{lc}
\hline \multicolumn{1}{c}{ NTM species } & Number of isolates \\
\hline MAC & 237 \\
M. gordonae & 43 \\
M. peregrinum & 33 \\
M. chelonae & 26 \\
M. kansasii & 13 \\
M. abscessus & 12 \\
M. scrofulaceum & 8 \\
M. xenopi & 2 \\
M. malmoense & 1 \\
M. mucogenicum & 1 \\
M. lentiflavum & 1 \\
M. fortuitum & 1 \\
M. spp. & 29 \\
TOTAL & 407 \\
\hline
\end{tabular}

NTM: Nontuberculous mycobacteria; and MAC: Mycobacterium avium complex.

(0.5\%). The number of isolates increased each year, from 52 in 2008 to 58 in 2009, 74 in 2010, 86 in 2011, and 137 in 2012. Of the 202 patients, $36(18 \%)$ were submitted to treatment for NTM-related lung disease, and 32 (89\%) of those met the current ATS/IDSA criteria for the diagnosis. Among the 36 patients treated, the mean age was 62 years (range, 32-89 years) and $22(61 \%)$ were male. The most common lung disease was COPD, seen in 12 (33\%) of the 36 patients, followed by bronchiectasis, in 9 (25\%), and tuberculosis sequelae, in 8 (22\%). There were 31 patients $(86.1 \%)$ presenting with cough, 29 $(80.6 \%)$ presenting with sputum production, and $23(63.9 \%)$ presenting with dyspnea. The main radiological findings were bronchiectasis, nodules, and micronodules. Among the 36 patients treated, the NTM identified were MAC in $34(94 \%), M$. kansasii in 1 (3\%), and M. xenopi in 1 (3\%). All patients were treated according to the ATS/IDSA statement recommendations. ${ }^{(3)}$ The 34 patients with MAC lung disease were treated with rifampin, ethambutol, and a macrolide (clarithromycin or azithromycin). The patient with $M$. kansasii infection was treated with rifampin, isoniazid, and ethambutol. The patient with $M$. xenopi infection was treated with rifampin, ethambutol, and clarithromycin. After the completion of treatment, relapse occurred in $5(13.9 \%)$ of the 34 patients with MAC infection, and 4 of those 5 had met the ATS/IDSA criteria. In three of those cases, the decision was made to submit the patients to retreatment -1 was cured, 1 died from lung cancer while still under treatment, and 1 is still under treatment at this writing-and in another case, the decision was not to treat, because the patient had been diagnosed with advanced cancer. There was no relapse in either of the patients treated for infection with M. kansasii and M. xenopi, respectively. In 2 (5.6\%) of the 36 patients, there was reinfection with other species (M. abscessus and $M$. scrofulaceum, respectively) after the completion of treatment, and both of those patients are still under treatment at this writing.

In Portugal, epidemiological studies are scarce. In 2008, Marinho et al. ${ }^{(4)}$ reported on 102 non-HIVinfected patients with NTM isolated from the respiratory system at our hospital over a 7-year period (from 1997 to 2004). In that study, the majority of the patients were male, the median age was 63 years, and most had an underlying lung disease, mainly tuberculosis sequelae or bronchiectasis. The authors reported that 16 (15.7\%) of the patients were treated for lung disease, and that $14(88 \%)$ of those patients met the ATS criteria in use at the time. ${ }^{(4)}$ In the present study, we identified nearly twice as many such patients over a period of only 4 years. The demographic profile of our sample was similar to that of the sample evaluated by Marinho et al. ${ }^{(4)}$ In our study, the most common lung diseases were COPD and bronchiectasis. Although the criteria used in order to define lung disease differed between the two studies, the proportion of patients treated and of those that met the respective ATS criteria were quite similar. In our study, the NTM most frequently isolated were MAC, which also had the greatest clinical relevance. Other studies conducted in Portugal have also reported MAC as the most frequently isolated NTM. ${ }^{(4-6)}$ In a recent global epidemiological study, ${ }^{(5)}$ the most frequently isolated NTM were $M$. avium, $M$. gordonae, $M$. xenopi, and $M$. fortuitum. In another hospital-based study, ${ }^{(7)} 16 \%$ of the patients with NTM isolated from respiratory specimens met the current ATS/ IDSA criteria.

The rapid increase in the incidence of NTM infection worldwide in recent years is probably due to the combination of growing awareness; improved culturing and molecular techniques for the detection of NTM; an aging population; and the increased prevalence of chronic lung disease and of diseases that result in immunosuppression. ${ }^{(1,2)} \ln$ addition, some 
authors have suggested that the decline in the incidence of tuberculosis has decreased the crossprotective mycobacterial immunity associated with M. tuberculosis infection, which has somehow promoted an increase in the incidence of NTM infection. ${ }^{(2)}$

Our study has some limitations. Because our data were collected only from patients seen in a pulmonology department, they might not be representative of the general population. What our results clearly indicate is that NTM is an emerging health problem at our hospital and possibly in the northern region of Portugal. Although a hospital-based study to confirm these results and larger-scale studies to evaluate the magnitude of the problem in the region would be advisable, clinicians need to be aware of the possibility of NTM infection, which can have devastating consequences for patients if not diagnosed and treated properly.

\section{References}

1. Johnson MM, Odell JA. Nontuberculous mycobacterial pulmonary infections. J Thorac Dis. 2014;6(3):210-20. http://dx.doi.org/10.3978/j.issn.2072-1439.2013.12.24
2. Kendall BA, Winthrop KL. Update on the epidemiology of pulmonary nontuberculous mycobacterial infections. Semin Respir Crit Care Med. 2013;34(1):87-94. http:// dx.doi.org/10.1055/s-0033-1333567

3. Griffith DE, Aksamit T, Brown-Elliott BA, Catanzaro A, Daley C, Gordin F, et al. An official ATS/IDSA statement: diagnosis, treatment, and prevention of nontuberculous mycobacterial diseases. Am J Respir Crit Care Med. 2007;175(4):367-416. http://dx.doi. org/10.1164/rccm.200604-571ST

4. Marinho A, Fernandes G, Carvalho T, Pinheiro D, Gomes 1. Nontuberculous mycobacteria in non-AIDS patients. Rev Port Pneumol. 2008;14(3):323-37. http://dx.doi. org/10.1016/S0873-2159(15)30241-5

5. Hoefsloot W, van Ingen J, Andrejak C, Angeby K, Bauriaud R, Bemer P, et al. The geographic diversity of nontuberculous mycobacteria isolated from pulmonary samples: an NTM-NET collaborative study. Eur Respir J. 2013;42(6):1604-13. http://dx.doi. org/10.1183/09031936.00149212

6. Amorim A, Macedo R, Lopes A, Rodrigues 1, Pereira E. Non-tuberculous mycobacteria in HIV-negative patients with pulmonary disease in Lisbon, Portugal. Scand J Infect Dis. 2010;42(8):626-8. http://dx.doi. org/10.3109/00365541003754485

7. Panagiotou M, Papaioannou Al, Kostikas K, Paraskeua M, Velentza E, Kanellopoulou M, et al. The epidemiology of pulmonary nontuberculous mycobacteria: data from a general hospital in Athens, Greece, 2007-2013. Pulm Med. 2014;2014:894976. http://dx.doi.org/10.1155/2014/894976 